\title{
Topological-antitopological fusion equations,
}

\section{pluriharmonic maps and special Kähler manifolds}

\author{
Vicente Cortés ${ }^{1}$ and Lars Schäfer ${ }^{1,2}$ \\ ${ }^{1}$ Institut Élie Cartan de Mathématiques, Université Henri Poincaré - Nancy 1, B.P. 239, \\ F-54506 Vandœuvre-lès-Nancy Cedex, France \\ cortes@iecn.u-nancy.fr, schafer@iecn.u-nancy.fr \\ 2 Mathematisches Institut der Universität Bonn, Beringstraße 1, D-53115 Bonn, Germany \\ schaefer@math.uni-bonn.de
}

March 31, 2004

\begin{abstract}
We introduce the notion of a $\mathrm{tt}^{*}$-bundle. It provides a simple definition, purely in terms of real differential geometry, for the geometric structures which are solutions of a general version of the equations of topological-antitopological fusion considered by Cecotti-Vafa, Dubrovin and Hertling. Then we give a simple characterization of the tangent bundles of special complex and special Kähler manifolds as particular types of $\mathrm{tt}^{*}$-bundles. We illustrate the relation between metric tt*-bundles of rank $r$ and pluriharmonic maps into the pseudo-Riemannian symmetric space $\mathrm{GL}(r) / \mathrm{O}(p, q)$ in the case of a special Kähler manifold of signature $(p, q)=(2 k, 2 l)$. It is shown that the pluriharmonic map coincides with the dual Gauß map, which is a holomorphic map into the pseudo-Hermitian symmetric space $\mathrm{Sp}\left(\mathbb{R}^{2 n}\right) / \mathrm{U}(k, l) \subset \mathrm{SL}(2 n) / \mathrm{SO}(p, q) \subset \mathrm{GL}(2 n) / \mathrm{O}(p, q)$, where $n=k+l$.
\end{abstract}

This work was supported by the 'Schwerpunktprogramm Stringtheorie' of the Deutsche Forschungsgemeinschaft. Research of L. S. was supported by a joint grant of the 'Deutscher Akademischer Austauschdienst' and the CROUS Nancy-Metz. 


\section{Contents}

1 tt*-equations and pluriharmonic maps 1

$\begin{array}{lll}2 & \text { Special complex and special Kähler manifolds } & 7\end{array}$

3 Special complex and special Kähler manifolds as solutions of the tt*equations

4 The pluriharmonic map in the case of a special Kähler manifold

4.1 The Gauß maps of a special Kähler manifold . . . . . . . . . . . . . . . . . 10

4.2 Holomorphic coordinates on the Grassmannian $G r_{0}^{k, l}\left(\mathbb{C}^{2 n}\right)$ of complex Lagrangian subspaces of signature $(k, l) \ldots \ldots \ldots 11$

4.3 The special Kähler metric in affine coordinates . . . . . . . . . . . . . 13

\section{$1 \quad \mathrm{tt}^{*}$-equations and pluriharmonic maps}

Definition 1 Att*-bundle $(E, D, S)$ over a complex manifold $(M, J)$ is a real vector bundle $E \rightarrow M$ endowed with a connection $D$ and a section $S \in \Gamma\left(T^{*} M \otimes\right.$ End $\left.E\right)$ which satisfy the $\mathrm{tt}^{*}$-equation

$$
R^{\theta}=0 \text { for all } \theta \in \mathbb{R},
$$

where $R^{\theta}$ is the curvature tensor of the connection $D^{\theta}$ defined by

$$
D_{X}^{\theta}:=D_{X}+(\cos \theta) S_{X}+(\sin \theta) S_{J X} \quad \text { for all } \quad X \in T M .
$$

$A$ metric tt*-bundle $(E, D, S, g)$ is a $t t^{*}$-bundle $(E, D, S)$ endowed with a possibly indefinite $D$-parallel fibre metric $g$ such that for all $p \in M$

$$
g\left(S_{X} Y, Z\right)=g\left(Y, S_{X} Z\right) \quad \text { for all } \quad X, Y, Z \in T_{p} M .
$$

$A$ unimodular metric $\mathrm{tt}^{*}$-bundle $(E, D, S, g)$ is a metric $t t^{*}$-bundle $(E, D, S, g)$ such that $\operatorname{tr} S_{X}=0$ for all $X \in T M$. An oriented unimodular metric tt*-bundle $(E, D, S, g$, or $)$ is a unimodular metric $t t^{*}$-bundle endowed with an orientation or of the bundle E.

Remarks: 1) In special cases, particularly emphasized in the literature, such as the moduli spaces of topological quantum field theories $[\mathrm{CV}, \mathrm{D}]$ and the moduli spaces of singularities $[\mathrm{H}]$, the complexified $\mathrm{tt}^{*}$-bundle $E^{\mathbb{C}}$ is identified with $T^{1,0} M$ and the metric $g$ is positive definite. Here we will consider the case $E=T M$, and hence $E^{\mathbb{C}}=T^{1,0} M+$ $T^{0,1} M$. This includes special complex and special Kähler manifolds, as we shall see.

2) If $(E, D, S)$ is a $\mathrm{tt}^{*}$-bundle then $\left(E, D, S^{\theta}\right)$ is a $\mathrm{tt}^{*}$-bundle for all $\theta \in \mathbb{R}$, where

$$
S^{\theta}:=D^{\theta}-D=(\cos \theta) S+(\sin \theta) S_{J} .
$$


The same remark applies to metric $t t^{*}$-bundles.

3) Notice that an oriented unimodular metric tt*-bundle $(E, D, S, g$, or $)$ carries a canonical metric volume element $\nu \in \Gamma\left(\wedge^{r} E^{*}\right), r=\mathrm{rk} E$, determined by $g$ and or, which is $D^{\theta}$ parallel for all $\theta \in \mathbb{R}$.

Proposition 1 Let $E \rightarrow M$ be a real vector bundle over a complex manifold $(M, J)$ such that $E$ is endowed with a connection $D$ and a section $S \in \Gamma\left(T^{*} M \otimes\right.$ End $\left.E\right)$. Then $(E, D, S)$ is a $t^{*}$-bundle if and only if the following equations are satisfied

(i) $d^{D} S=d^{D} S_{J}=0$, where $S$ and $S_{J}$ are considered as one-forms with values in End $E$ and $d^{D}$ is the exterior covariant derivative defined by $D$,

(ii) $\left[S_{X}, S_{Y}\right]=\left[S_{J X}, S_{J Y}\right]$ for all $X$ and $Y$,

(iii) $R^{D}(X, Y)+\left[S_{X}, S_{Y}\right]=0$ for all $X$ and $Y$.

Proof: Using the relations $2 \cos \theta \sin \theta=\sin 2 \theta, 2 \cos ^{2} \theta=1+\cos 2 \theta$ and $2 \sin ^{2} \theta=$ $1-\cos 2 \theta$, we obtain a (finite) Fourier decomposition of $R^{D^{\theta}}$ in the variable $\theta$. The tt*-equation $R^{D^{\theta}}=0$ shows that all Fourier components are zero. This yields (i-iii).

Definition 2 Let $(M, J)$ be a complex manifold and $(N, h)$ a pseudo-Riemannian manifold. A map $f: M \rightarrow N$ is called pluriharmonic if $\left.f\right|_{C}$ is harmonic for all complex curves $C \subset M$.

Notice that the harmonicity of $\left.f\right|_{C}$ is independent of the choice of a Riemannian metric in the conformal class of $C$, by conformal invariance of the harmonic map equation for (real) surfaces.

Proposition 2 Let $(M, J)$ be a complex manifold and $(N, h)$ a pseudo-Riemannian manifold with Levi-Civita connection $\nabla^{h}, D$ a connection on $M$ which satisfies

$$
D_{J Y} X=J D_{Y} X
$$

for all vector fields which satisfy $\mathcal{L}_{X} J=0$ (i.e. for which $X-i J X$ is holomorphic) and $\nabla$ the connection on $T^{*} M \otimes f^{*} T N$ which is induced by $D$ and $\nabla^{h}$.

(i) A map $f: M \rightarrow N$ is pluriharmonic if and only if it satisfies the following equation

$$
\nabla^{\prime \prime} \partial f=0
$$

where $\partial f=d f^{1,0} \in \Gamma\left(\bigwedge^{1,0} T^{*} M \otimes_{\mathbb{C}}(T N)^{\mathbb{C}}\right)$ is the $(1,0)$-component of $d f$ and $\nabla^{\prime \prime}$ is the $(0,1)$-component of $\nabla=\nabla^{\prime}+\nabla^{\prime \prime}$.

(ii) Any complex manifold $(M, J)$ admits a torsion-free complex connection, i.e. a torsionfree connection $D$ which satisfies $D J=0$.

(iii) Any torsion-free complex connection D satisfies (1.5). 
Proof: (i) The condition (1.5) means that $D^{\prime \prime} Z=0$ for all local holomorphic vector fields $Z$, i.e. $\Gamma_{\bar{\alpha} \beta}^{\gamma}=\Gamma_{\bar{\alpha} \beta}^{\bar{\gamma}}=0$ in terms of the Christoffel symbols of $D$ with respect to holomorphic coordinates $z^{\alpha}$. This implies that the Christoffel symbols of $D$ do not contribute to the equation (1.6). The equation is therefore independent of the choice of connection $D$. In fact, it is straightforward to check that the restriction of (1.6) to every complex curve $C$ reduces to the harmonic map equation for $\left.f\right|_{C}: C \rightarrow N$.

(ii) is well known, see $[\mathrm{KN}]$.

(iii) The conditions $T^{D}=0$ and $D J=0$ imply that

$$
D_{J Y} X-J D_{Y} X=[J Y, X]+D_{X}(J Y)-J D_{Y} X=[J Y, X]+J[X, Y]=-\left(\mathcal{L}_{X} J\right) Y .
$$

The right-hand side vanishes if $\mathcal{L}_{X} J=0$.

Given a Hermitian metric $\gamma$ on $T^{1,0} M$, or, more generally, a pseudo-Hermitian metric, the Chern connection of $\gamma$ is the unique Hermitian connection $\mathcal{D}$ in the holomorphic bundle $T^{1,0} M$ which satisfies $\mathcal{D}^{\prime \prime} Z=0$ for all holomorphic local sections $Z$ of $T^{1,0} M$. The last property is usually written as $\mathcal{D}^{\prime \prime}=\bar{\partial}$.

Proposition 3 Let $(M, J)$ be a complex manifold and $\mathcal{D}$ the Chern connection of a pseudo-Hermitian metric $\gamma$ on $T^{1,0} M$. Then there is a unique connection $D$ in the real tangent bundle $T M$ such that $D Z=\mathcal{D} Z$ for all local sections $Z$ of $T^{1,0} M$, where $D$ has been complex bilinearly extended to a connection on the complexified tangent bundle. The connection $D$ satisfies (1.5), DJ $=0$ and $D g=0$, where $g$ is the J-invariant pseudoRiemannian metric defined by

$$
g(X, X)=2 \gamma\left(X^{1,0}, X^{1,0}\right), \quad X^{1,0}:=\frac{1}{2}(X-i J X),
$$

for all $X \in T M$.

Conversely, let $g$ be a J-invariant pseudo-Riemannian metric on a complex manifold $(M, J)$. Then there exists a unique connection $D$ in $T M$, which satisfies the conditions (1.5), $D J=0$ and $D g=0$. Moreover, $D$ induces a connection in $T^{1,0} M$, which is the Chern connection of the pseudo-Hermitian metric $\gamma$ on $T^{1,0} M$ defined by (1.8).

The factor 2 is chosen such that $\gamma$ coincides with the restriction to $T^{1,0} M$ of the sesquilinear extension of $g$ to the complexified tangent bundle.

Proof: We define a connection $D$ in the complexified tangent bundle $(T M)^{\mathbb{C}}$ by

$$
D_{X} Z:=\mathcal{D}_{X} Z \text { and } D_{X} \bar{Z}:=\overline{\mathcal{D}_{\bar{X}} Z}
$$

for all local sections $X$ of $(T M)^{\mathbb{C}}$ and $Z$ of $T^{1,0} M$. By construction $D$ is real, i.e. is the complex bilinear extension of a connection in $T M$, which we denote by the same symbol $D$. Obviously, it is the only real connection such that $D Z=\mathcal{D} Z$ for all local sections $Z$ of $T^{1,0} M$. The equation (1.5) follows from $\mathcal{D}^{\prime \prime}=\bar{\partial}$. By construction, $D$ preserves the decomposition $(T M)^{\mathbb{C}}=T^{1,0} M+T^{0,1} M$. Therefore, $D J=0$. Finally, $D g=0$ follows from the fact that $\mathcal{D}$ is Hermitian.

Conversely, let $(M, J, g)$ be a pseudo-Hermitian manifold. Then we can define a pseudo-Hermitian metric $\gamma$ in $T^{1,0} M$ by (1.8) and consider its Chern connection $\mathcal{D}$. As 
we know, it induces a connection $D$ in $T M$ which satisfies (1.5), $D J=0$ and $D g=0$. To prove the uniqueness, let $\tilde{D}$ be an other connection satisfying (1.5), $\tilde{D} J=0$ and $\tilde{D} g=0$. $\tilde{D}$ induces a connection $\tilde{\mathcal{D}}$ in $T^{1,0} M$, which satisfies $\tilde{\mathcal{D}}^{\prime \prime}=\bar{\partial}$, due to (1.5), and which is Hermitian with respect to $\gamma$. Therefore, $\tilde{\mathcal{D}}$ is the Chern connection of $\gamma$, i.e. $\tilde{\mathcal{D}}=\mathcal{D}$. This implies $D=\tilde{D}$, by the first part of the proof.

Given a metric tt*-bundle $(E, D, S, g)$, we consider the flat connection $D^{\theta}$ for $\theta=0$ : $\nabla:=D^{0}$. Any parallel frame $s=\left(s_{1}, \ldots, s_{r}\right)$ of $E$ with respect to $\nabla$ defines a map

$$
\begin{aligned}
& G=G^{(s)}: M \rightarrow \operatorname{Sym}_{p, q}\left(\mathbb{R}^{r}\right)=\left\{A \in \mathrm{GL}(r) \mid A^{t}=A \text { has signature }(p, q)\right\} \\
& x \mapsto G(x):=\left(g_{x}\left(s_{i}(x), s_{j}(x)\right)\right),
\end{aligned}
$$

where $(p, q)$ is the signature of the metric $g$.

Similarly, for an oriented unimodular metric tt*-bundle $(E, D, S, g, \nu)$ with canonical volume element $\nu$ and a $\nabla$-parallel frame $s=\left(s_{1}, \ldots, s_{r}\right)$ such that $\nu\left(s_{1}, s_{2}, \ldots, s_{r}\right)=1$ we have a map

$$
G=G^{(s)}: M \rightarrow \operatorname{Sym}_{p, q}^{1}\left(\mathbb{R}^{r}\right)=\left\{A \in \operatorname{Sym}_{p, q}\left(\mathbb{R}^{r}\right) \mid \operatorname{det} A=(-1)^{q}\right\} .
$$

By Sylvester's Theorem, the general linear group GL $(r)$ acts transitively on the manifold $\operatorname{Sym}_{p, q}\left(\mathbb{R}^{r}\right)$, which we can identify with the pseudo-Riemannian symmetric space

$$
S(p, q):=\mathrm{GL}(r) / \mathrm{O}(p, q) \text {. }
$$

The subgroup $\mathrm{O}(p, q) \subset \mathrm{GL}(r)$ is the stabilizer of the matrix $I_{p, q}=\operatorname{diag}\left(\mathbb{1}_{p},-\mathbb{1}_{q}\right)$. We shall identify the tangent space of the coset space $S(p, q)$ at the canonical base point $o=e \mathrm{O}(p, q)$ with the vector space

$$
\operatorname{sym}(p, q):=\{A \in \mathfrak{g l}(r) \mid \eta(A \cdot, \cdot)=\eta(\cdot, A \cdot)\}
$$

of symmetric endomorphisms of $\mathbb{R}^{r}$ with respect to the standard scalar product $\eta=\eta_{p, q}$ of signature $(p, q)$, which is represented by the matrix $I_{p, q}$. The structure of a symmetric space is defined by the symmetric decomposition

$$
\mathfrak{g l}(r)=\mathfrak{o}(p, q)+\operatorname{sym}(p, q) .
$$

The pseudo-Riemannian metric is defined by an $\mathrm{O}(p, q)$-invariant pseudo-Euclidean scalar product on $\operatorname{sym}(p, q)$. For instance, we may choose the metric induced by the trace form:

$$
\mathfrak{g l}(r) \ni(X, Y) \mapsto \operatorname{tr} X Y
$$

Similarly, $\operatorname{SL}(r)$ acts transitively on the manifold $\operatorname{Sym}_{p, q}^{1}\left(\mathbb{R}^{r}\right)$, which we can identify with the pseudo-Riemannian symmetric space

$$
S_{1}(p, q):=\mathrm{SL}(r) / \mathrm{SO}(p, q) .
$$

We have the de Rham decomposition

$$
S(p, q)=\mathbb{R} \times S_{1}(p, q),
$$


where the flat factor corresponds to the connected central subgroup

$$
\mathbb{R}^{>0}=\{\lambda \mathrm{Id} \mid \lambda>0\} \subset \mathrm{GL}(r)
$$

and the other factor is always indecomposable and even irreducible if $(p, q) \neq(1,1)$. The tangent space of $\mathrm{SL}(r) / \mathrm{SO}(p, q)$ at the canonical base point $o=e \mathrm{SO}(p, q)$ is identified with the trace-free $\eta$-symmetric matrices:

$$
\operatorname{sym}_{0}(p, q):=\{A \in \operatorname{sym}(p, q) \mid \operatorname{tr} A=0\} .
$$

Under a change of parallel (respectively, parallel unimodular) frame $s \rightarrow s u, u \in \mathrm{GL}(r)$ (respectively, $u \in \mathrm{SL}(r)$ ), the map $G=G^{(s)}$ transforms as

$$
G^{(s u)}=u^{-1} \cdot G^{(s)}=u^{t} G^{(s)} u
$$

where the dot stands for the action of $\mathrm{GL}(r)$ on $\operatorname{Sym}_{p, q}\left(\mathbb{R}^{r}\right)$.

The following theorem is proven in [S2], cf. [S1]. In the case where $E^{\mathbb{C}}=T^{1,0} M$ and the metric $g$ is positive definite it is due to Dubrovin [D].

Theorem 1 Let $(E, D, S, g)$ be a metric $t t^{*}$-bundle over a simply connected complex manifold $M$. Then the map

$$
G^{(s)}=\left(g\left(s_{i}, s_{j}\right)\right): M \rightarrow \operatorname{Sym}_{p, q}\left(\mathbb{R}^{r}\right) \cong \mathrm{GL}(r) / \mathrm{O}(p, q)=S(p, q)
$$

associated to a parallel frame $s=\left(s_{1}, \ldots, s_{r}\right)$ of $E$ with respect to the flat connection $\nabla=D^{0}$ is pluriharmonic. Moreover, for all $x \in M$, the image of $T_{x}^{1,0} M \subset\left(T_{x} M\right) \otimes \mathbb{C}$ under the complex linear extension of $d L_{u}^{-1} d G_{x}: T_{x} M \rightarrow T_{o} S(p, q)=\operatorname{sym}(p, q)$ consists of commuting matrices, where $u \in \mathrm{GL}(r)$ is any element such that $G(x)=u \cdot o$ and $L_{u}: S(p, q) \rightarrow S(p, q)$ is the isometry of $S(p, q)$ induced by the left-multiplication by $u$ in $\mathrm{GL}(r)$.

Conversely, let $M$ be a simply connected complex manifold and $f: M \rightarrow \operatorname{Sym}_{p, q}\left(\mathbb{R}^{r}\right) \cong$ $S(p, q)$ a pluriharmonic map such that, for all $x \in M$, the image of $T_{x}^{1,0} M$ under the complex linear extension of $d L_{u}^{-1} d f_{x}: T_{x} M \rightarrow T_{o} S(p, q)=\operatorname{sym}(p, q)$ consists of commuting matrices, where $u \in \mathrm{GL}(r)$ is any element such that $f(x)=u \cdot o$. Then there exists a metric $t t^{*}$-bundle $(E, D, S, g)$ over $M$ and a parallel frame $s$ such that $f=G^{(s)}$. The condition on the image of $T_{x}^{1,0} M$ is automatically satisfied if $p q=0$, which corresponds to a positive or negative definite metric $g$.

The same correspondence holds for oriented unimodular $t t^{*}$-bundles and pluriharmonic maps into $\operatorname{Sym}_{p, q}^{1}\left(\mathbb{R}^{r}\right) \cong \mathrm{SL}(r) / \mathrm{SO}(p, q)=S_{1}(p, q)$.

Now we shall explain in more detail the condition on the image of $T^{1,0} M$ under the differential of $f$ in the theorem. Above we have always identified $\operatorname{Sym}_{p, q}\left(\mathbb{R}^{r}\right)$ with $S(p, q)$. Let us denote by

$$
\varphi: \operatorname{Sym}_{p, q}\left(\mathbb{R}^{r}\right) \rightarrow S(p, q), \quad S \mapsto \tilde{S}=\varphi(S),
$$

that identification, which is $\mathrm{GL}(r)$-equivariant and maps $I=I_{p, q}$ to the canonical base point $o$. We can identify the tangent space $T_{S} \operatorname{Sym}_{p, q}\left(\mathbb{R}^{r}\right)$ at $S \in \operatorname{Sym}_{p, q}\left(\mathbb{R}^{r}\right)$ with the (ambient) vector space of symmetric matrices:

$$
T_{S} \operatorname{Sym}_{p, q}\left(\mathbb{R}^{r}\right)=\operatorname{Sym}\left(\mathbb{R}^{r}\right):=\left\{A \in \operatorname{Mat}(r, \mathbb{R}) \mid A^{t}=A\right\} .
$$


As above for $S=I$, the tangent space $T_{\tilde{S}} S(p, q)$ is canonically identified with the vector space of $S$-symmetric matrices:

$$
T_{\tilde{S}} S(p, q)=\operatorname{sym}(S):=\left\{A \in \mathfrak{g l}(r) \mid A^{t} S=S A\right\} .
$$

Note that $\operatorname{sym}\left(I_{p, q}\right)=\operatorname{sym}(p, q)$.

Proposition 4 The differential of $\varphi$ at $S \in \operatorname{Sym}_{p, q}\left(\mathbb{R}^{r}\right)$ is given by

$$
\operatorname{Sym}\left(\mathbb{R}^{r}\right) \ni X \mapsto-\frac{1}{2} S^{-1} X \in S^{-1} \operatorname{Sym}\left(\mathbb{R}^{r}\right)=\operatorname{sym}(S) .
$$

Let us now consider the differential

$$
d f_{x}: T_{x} M \rightarrow \operatorname{Sym}\left(\mathbb{R}^{r}\right)
$$

of $f: M \rightarrow \operatorname{Sym}_{p, q}\left(\mathbb{R}^{r}\right)$ at $x \in M$ and the differential

$$
d \tilde{f}_{x}: T_{x} M \rightarrow \operatorname{sym}(f(x))
$$

of $\tilde{f}=\varphi \circ f: M \rightarrow S(p, q)$. Then the condition on the image of the differential of $f$ in the theorem is that

$$
d L_{u}^{-1} d \tilde{f}\left(T_{x}^{1,0} M\right) \subset \operatorname{sym}(p, q) \otimes \mathbb{C} \quad \text { consists of commuting matrices },
$$

where $\tilde{f}(x)=u o$. This is equivalent to the condition that $d \tilde{f}\left(T_{x}^{1,0} M\right) \subset \operatorname{sym}(\tilde{f}(x)) \otimes \mathbb{C}$ consists of commuting matrices. This follows from the fact that

$$
d L_{u}: T_{o} S(p, q) \rightarrow T_{u o} S(p, q)=T_{\tilde{f}(x)} S(p, q)
$$

corresponds to

$$
A d_{u}: \operatorname{sym}(p, q)=\operatorname{sym}(I) \rightarrow \operatorname{sym}(u \cdot I)=\operatorname{sym}(\tilde{f}(x))
$$

and that the adjoint representation preserves the Lie bracket.

Finally, $d \tilde{f}_{x}=d \varphi d f_{x}=-\frac{1}{2} f(x)^{-1} d f_{x}$ and, therefore,

$$
d \tilde{f}\left(T_{x}^{1,0} M\right)=f(x)^{-1} d f_{x}\left(T_{x}^{1,0} M\right) .
$$

This shows that $f$ satisfies the condition (1.28) if and only if the matrices $f(x)^{-1} d f_{x}(Z)$ and $f(x)^{-1} d f_{x}(W)$ commute for all $Z, W \in T_{x}^{1,0} M$. This is equivalent to

$$
\left[f(x)^{-1} d f_{x}(J X), f(x)^{-1} d f_{x}(J Y)\right]=\left[f(x)^{-1} d f_{x}(X), f(x)^{-1} d f_{x}(Y)\right]
$$

for all $X, Y \in T_{x} M$. 


\section{Special complex and special Kähler manifolds}

In this section we recall some basic results on special complex manifolds and special Kähler manifolds. For more detailed information the reader is referred to $[\mathrm{ACD}]$, see also $[\mathrm{F}]$.

Definition 3 A special complex manifold $(M, J, \nabla)$ is a complex manifold $(M, J)$ endowed with a flat torsion-free connection $\nabla$ (on the real tangent-bundle) such that $\nabla J$ is symmetric.

$A$ special Kähler manifold $(M, J, \nabla, \omega)$ is a special complex manifold $(M, J, \nabla)$ endowed with a J-invariant and $\nabla$-parallel symplectic form $\omega$. The (pseudo)-Kähler-metric $g(\cdot, \cdot)=$ $\omega(J \cdot, \cdot)$ is called the special Kähler metric of the special Kähler manifold $(M, J, \nabla, \omega)$.

Given a complex manifold $(M, J)$ with a flat connection $\nabla$, we define its conjugate connection by

$$
\nabla_{X}^{J}=\nabla_{X}-J \nabla_{X} J \text { with } X \in T M .
$$

On a special complex manifold $(M, J, \nabla)$ the connection $\nabla^{J}$ is torsion-free. In addition, one can introduce a torsion-free connection

$$
D:=\frac{1}{2}\left(\nabla+\nabla^{J}\right)=\nabla-S, \text { where } S:=\frac{1}{2} J \nabla J
$$

which satifies $D J=0$, as follows from a short calculation.

In the case of a special Kähler manifold $(M, J, \nabla, \omega)$ the connection $D$ is the Levi-Civita connection of the special Kähler metric $g$ and the endomorphism-field $S$ anticommutes with the complex structure $J$, i.e. :

$$
J S_{X}=-S_{X} J \text { for all } \quad X \in T M .
$$

Now we explain part of the extrinsic construction of special Kähler-manifolds given in $[\mathrm{ACD}]$. In order to do this, we consider the complex vector space $V=T^{*} \mathbb{C}^{n}=\mathbb{C}^{2 n}$ with canonical coordinates $\left(z^{1}, \ldots, z^{n}, w_{1}, \ldots, w_{n}\right)$ endowed with the standard complex symplectic form $\Omega=\sum_{i=1}^{n} d z^{i} \wedge d w_{i}$ and the standard real structure $\tau: V \rightarrow V$ with fixed points $V^{\tau}=T^{*} \mathbb{R}^{n}$. These define a Hermitian form $\gamma:=i \Omega(\cdot, \tau \cdot)$.

Let $(M, J)$ be a complex manifold $(M, J)$ of complex dimension $n$. We call a holomorphic immersion $\phi: M \rightarrow V$ nondegenerate (respectively Lagrangian) if $\phi^{*} \gamma$ is nondegenerate (respectively, if $\phi^{*} \Omega=0$ ). If $\phi$ is nondegenerate it defines a, possibly indefinite, Kähler metric $g=\operatorname{Re} \phi^{*} \gamma$ on the complex manifold $(M, J)$ and the corresponding Kähler form $g(\cdot, J \cdot)$ is a $J$-invariant symplectic form.

The following theorem gives a description of simply connected special Kähler-manifolds in terms of the above data:

Theorem 2 [ACD] Let $(M, J, \nabla, \omega)$ be a simply connected special Kähler manifold of complex dimension $n$, then there exists a holomorphic nondegenerate Lagrangian immersion $\phi: M \rightarrow V=T^{*} \mathbb{C}^{n}$ inducing the Kähler metric $g$, the connection $\nabla$ and the symplectic form $\omega=2 \phi^{*}\left(\sum_{i=1}^{n} d x^{i} \wedge d y_{i}\right)=g(\cdot, J \cdot)$ on $M$. Moreover, $\phi$ is unique up to an affine transformation of $V$ preserving the complex symplectic form $\Omega$ and the real structure $\tau$. The flat connection $\nabla$ is completely determined by the condition $\nabla \phi^{*} d x^{i}=\nabla \phi^{*} d y_{i}=0$, $i=1, \ldots, n$, where $x^{i}=\operatorname{Re} z^{i}$ and $y_{i}=\operatorname{Re} w_{i}$. 


\section{Special complex and special Kähler manifolds as solutions of the $\mathrm{tt}^{*}$-equations}

Let $(E, D, S)$ be a $\mathrm{tt}^{*}$-bundle over a complex manifold $(M, J)$. We are now interested in the case $E=T M$. In that case it is natural to consider tt*-bundles for which the connection $D^{\theta}=D+(\cos \theta) S+(\sin \theta) S_{J}$ is torsion-free.

Definition 4 A tt*-bundle $(T M, D, S)$ over a complex manifold $(M, J)$ is called special if $D^{\theta}$ is torsion-free and special, i.e. $D^{\theta} J$ is symmetric for all $\theta$.

Proposition $5 \quad A t t^{*}$-bundle $(T M, D, S)$ is special if and only if $D$ is torsion-free and $D J, S$ and $S_{J}$ are symmetric.

Proof: The torsion $T^{\theta}$ of $D^{\theta}$ is given by

$$
T^{\theta}(X, Y)=T(X, Y)+\cos \theta\left(S_{X} Y-S_{Y} X\right)+\sin \theta\left(S_{J X} Y-S_{J Y} X\right),
$$

where $T$ is the torsion of $D$. This shows that $T^{\theta}=0$ for all $\theta$ if and only if $T=0$ and $S$ and $S_{J}$ are symmetric. The equation

$$
\left(D_{X}^{\theta} J\right) Y=\left(D_{X} J\right) Y+\cos \theta\left[S_{X}, J\right] Y+\sin \theta\left[S_{J X}, J\right] Y
$$

shows that $D^{\theta} J$ is symmetric if $D J, S$ and $S_{J}$ are symmetric. Conversely, if $T^{\theta}=0$ and $D^{\theta} J$ is symmetric, then, by the first part of the proof, $S$ and $S_{J}$ are symmetric and equation (3.2) shows that $D J$ is symmetric.

\section{Theorem 3}

(i) Let $(M, J, \nabla)$ be a special complex manifold. Put $S:=\frac{1}{2} J \nabla J$ and $D:=\nabla-S$. Then $(T M, D, S)$ is a special $t t^{*}$-bundle, which satisfies the additional conditions:

a) $S_{X} J=-J S_{X}$ for all $X \in T M$ and

b) $D J=0$.

This defines a map $\Phi$ from special complex manifolds to special $t t^{*}$-bundles.

(ii) Let $(T M, D, S)$ be a special $t t^{*}$-bundle over a complex manifold $(M, J)$. Then $(M, J, \nabla:=D+S)$ is a special complex manifold. This defines a map $\Psi$ from special $t t^{*}$-bundles to special complex manifolds such that $\Psi \circ \Phi=\mathrm{Id}$. If $(T M, D, S)$ is a special tt*-bundle satisfying the conditions a) and b) in (i), then $\Phi(\Psi(T M, D, S))=$ $(T M, D, S)$.

(iii) Let $(M, J, g, \nabla)$ be a special Kähler manifold with $S$ and $D$ defined as in (i). Then $(T M, D, S, g)$ is a special metric $t t^{*}$-bundle. This defines a map $\Phi$ from special Kähler manifolds to special metric $t t^{*}$-bundles.

(iv) Let $(T M, D, S, g)$ be a special metric tt*-bundle over a pseudo-Hermitian manifold $(M, J, g)$ satisfying the conditions a) and b) in (i). Then $(M, J, \nabla:=D+S, g)$ is a special Kähler manifold. In particular, we have a map $\Psi$ from special metric tt*-bundles over pseudo-Hermitian manifolds satisfying a) and b) to special Kähler manifolds. Moreover, $\Psi$ is a bijection and $\Psi^{-1}=\Phi$. 
(v) Let $(T M, D, S, g)$ be a metric $t^{*}$-bundle over a pseudo-Hermitian manifold $(M, J, g)$ satisfying $a)$ and $b)$ in (i) and such that $D$ is torsion-free. Then it is special if and only if $(M, J, \nabla:=D+S, g)$ is a special Kähler manifold.

Proof: (i) Let $(M, J, \nabla)$ be a special complex manifold with $S$ and $D$ defined as above. Then

$$
\nabla^{\theta}=e^{\theta J} \circ \nabla \circ e^{-\theta J}
$$

is a family of flat torsion-free special connections. Using $\nabla=D+S$ and (2.3) we can write

$$
\nabla_{X}^{\theta}=D_{X}+e^{2 \theta J} S_{X}
$$

The following calculation shows that $\nabla^{\theta}=D^{-2 \theta}$, where $D^{\theta}$ is defined in (1.2):

$$
\begin{aligned}
\nabla_{X}^{\theta}-D_{X} & =e^{2 \theta J} S_{X}=\cos (2 \theta) S_{X}+\sin (2 \theta) J S_{X} \\
& \stackrel{(*)}{=} \cos (-2 \theta) S_{X}+\sin (-2 \theta) S_{J X}=D_{X}^{-2 \theta}-D_{X}, \quad X \in T M .
\end{aligned}
$$

At $(*)$ we have used that $J S_{X}=-S_{J X}$, which follows from

$$
J S_{X} Y=J S_{Y} X=-S_{Y} J X=-S_{J X} Y, \quad X, Y \in T_{p} M .
$$

Here we used the symmetry of $S$ and (2.3). This shows that $(T M, D, S)$ is a special tt*-bundle.

(ii) Let $(T M, D, S)$ be a special tt*-bundle. This means that $D^{\theta}$ is flat, torsion-free and special. In particular, $\nabla=D+S=D^{0}$ is flat, torsion-free and special and $(M, J, \nabla)$ is a special complex manifold. It is clear that $\Psi \circ \Phi=\mathrm{Id}$.

Conversely, let $(T M, D, S)$ be a special tt*-bundle such that $D J=0$ and $S_{X} J=-J S_{X}$ for all $X$. Then we can recover $D$ and $S$ from $\nabla=D+S$ by the formulas $S=\frac{1}{2} J \nabla J$ and $D=\nabla-S$. In fact, Let $\left(T M, D^{\prime}, S^{\prime}\right)$ be an other special tt*-bundle over $(M, J)$ such that $D^{\prime} J=0$ and $S_{X}^{\prime} J=-J S_{X}^{\prime}$ for all $X \in T M$ and $\nabla=D+S=D^{\prime}+S^{\prime}$. Then

$$
0=D_{X}^{\prime} J=\nabla_{X} J-\left[S_{X}^{\prime}, J\right]=\nabla_{X} J+2 J S_{X}^{\prime}
$$

for all $X \in T M$. This shows that $S_{X}^{\prime}=\frac{1}{2} J \nabla_{X} J=S_{X}$ and $D^{\prime}=\nabla-S^{\prime}=\nabla-S=D$.

(iii) Let $(M, J, g, \nabla)$ be a special Kähler manifold with $S$ and $D$ defined as in (i). Then, by (i), (TM,D,S) is a special tt*-bundle and satifies a) and b). To prove that it is a metric tt*-bundle we have to check that $D g=0$ and that (1.3) is satisfied. Since $D J=0$, by b), the equation $D g=0$ is equivalent to the following claim:

Claim: The Kähler form $\omega$ is $D$-parallel; $D \omega=0$.

In fact $\nabla \omega=0$ and $S_{X}=\frac{1}{2} J \nabla_{X} J, X \in T M$, is the product of two anticommuting $\omega$-skew-symmetric endomorphisms $A=\frac{1}{2} J$ and $B=\nabla_{X} J$. This implies that $S_{X}$ is $\omega$-skew-symmetric and, thus, $D \omega=0$.

The endomorphism $S_{X}$ is $\omega$-skew-symmetric and anticommutes with $J$, by a). Therefore $S_{X}$ is symmetric with respect to $g=\omega(J \cdot, \cdot)$. 
(iv) Let $(T M, D, S, g)$ be a special metric tt*-bundle over a pseudo-Hermitian manifold $(M, J, g)$ satisfying a) and b) in (i). Thanks to (ii), we know already that $(M, J, \nabla:=$ $D+S)$ is a special complex manifold. Therefore it suffices to prove that $\nabla \omega=0$. The assumption $D g=0$ and property b) imply that $D \omega=0$. Now it is sufficient to observe that the endomorphisms $S_{X}, X \in T M$, are $\omega$-skew-symmetric. In fact, $S_{X}$ is $g$-symmetric in virtue of (1.3) and anticommutes with $J$, by a). This shows that $(M, J, \nabla, g)$ is a special Kähler manifold. The remaining statements follow from (ii).

(v) Let $(T M, D, S, g)$ be a metric tt*-bundle over a pseudo-Hermitian manifold $(M, J, g)$ such that $(M, J, \nabla:=D+S, g)$ is a special Kähler manifold. If $D$ is torsion-free, then it is the Levi-Civita connection of $g$ and, thus, $D=\nabla-\frac{1}{2} J \nabla J$, see section 2. Now we can conclude that $\Phi(M, J, \nabla, g)=(T M, D, S, g)$. This shows that $(T M, D, S, g)$ is a special metric $t^{*}$-bundle. The converse follows from (iv).

Corollary 1 Any special metric tt*-bundle (TM,D,S,g) over a pseudo-Hermitian manifold $(M, J, g)$ which satisfies a) and b) in Theorem $3(i)$ is oriented and unimodular.

Proof: $T M$ is canonically oriented by the complex structure $J$. By Theorem $3,(M, J, g, \nabla$ $=D+S)$ is a special Kähler manifold. Its Kähler form is parallel with respect to $D$ and $\nabla$ and hence invariant under $S_{X}=\nabla_{X}-D_{X}$ for all $X \in T M$. This shows that $\operatorname{tr} S_{X}=0$.

In $[\mathrm{H}]$ special complex and special Kähler geometry is interpreted in terms of variations of Hodge structure of weight 1 on the complexified tangent bundle. From this interpretation and his discussion of $t^{*}$-geometry, it follows that any special complex (respectively, special Kähler) manifold defines a tt*-bundle (respectively, a metric tt*-bundle) in the sense of our definition.

\section{The pluriharmonic map in the case of a special Kähler manifold}

\subsection{The Gauß maps of a special Kähler manifold}

Let $(M, J, g, \nabla)$ be a special Kähler manifold of complex dimension $n=k+l$ and of Hermitian signature $(k, l)$, i.e. $g$ has signature $(2 k, 2 l)$. Let $(\widetilde{M}, J, g, \nabla)$ be its universal covering with the pullback special Kähler structure, which is again denoted by $(J, g, \nabla)$. According to Theorem 2, there exists a (holomorphic) Kählerian Lagrangian immersion $\phi: \widetilde{M} \rightarrow V=T^{*} \mathbb{C}^{n}=\mathbb{C}^{2 n}$, which is unique up to a complex affine transformation of $V$ with linear part in $\operatorname{Sp}\left(\mathbb{R}^{2 n}\right)$. We consider the dual Gauß map of $\phi$

$$
L: \widetilde{M} \rightarrow G r_{0}^{k, l}\left(\mathbb{C}^{2 n}\right), \quad p \mapsto L(p):=T_{\phi(p)} \widetilde{M}:=d \phi_{p} T_{p} \widetilde{M} \subset V
$$

into the Grassmannian of complex Lagrangian subspaces $W \subset V$ of signature $(k, l)$, i.e. such that the restriction of $\gamma$ to $W$ is a Hermitian form of signature $(k, l)$. The map $L: \widetilde{M} \rightarrow G r_{0}^{k, l}\left(\mathbb{C}^{2 n}\right)$ is in fact the dual of the Gauß map

$$
L^{\perp}: \widetilde{M} \rightarrow G r_{0}^{l, k}\left(\mathbb{C}^{2 n}\right), \quad p \mapsto L(p)^{\perp}=\overline{L(p)} \cong L(p)^{*} .
$$


Here $L(p)^{\perp}$ stands for the $\gamma$-orthogonal complement of $L(p)$ and the isomorphism $\overline{L(p)} \cong$ $L(p)^{*}$ is induced by the symplectic form $\Omega$ on $V=L(p) \oplus \overline{L(p)}$.

The Grassmannian $G r_{0}^{k, l}\left(\mathbb{C}^{2 n}\right)$ is an open subset of the complex Grassmannian $G r_{0}\left(\mathbb{C}^{2 n}\right)$ of complex Lagrangian subspaces $W \subset V$ and hence a complex submanifold.

Proposition 6 (i) The dual Gauß map $L: \widetilde{M} \rightarrow G r_{0}^{k, l}\left(\mathbb{C}^{2 n}\right)$ is holomorphic

(ii) The Gauß map $L^{\perp}: \widetilde{M} \rightarrow G r_{0}^{l, k}\left(\mathbb{C}^{2 n}\right)$ is antiholomorphic.

Proof: The holomorphicity of $L$ follows from that of $\phi$. Part (ii) follows from (i), since $L^{\perp}=\bar{L}: p \mapsto \overline{L(p)}$.

The real symplectic group $\operatorname{Sp}\left(\mathbb{R}^{2 n}\right)$ acts transitively on $G r_{0}^{k, l}\left(\mathbb{C}^{2 n}\right)$ and we have the following identification:

$$
G r_{0}^{k, l}\left(\mathbb{C}^{2 n}\right)=\operatorname{Sp}\left(\mathbb{R}^{2 n}\right) / \mathrm{U}(k, l) .
$$

Here $\mathrm{U}(k, l) \subset \mathrm{Sp}\left(\mathbb{R}^{2 n}\right)$ is defined as the stabilizer of

$$
W_{o}=\operatorname{span}\left\{\frac{\partial}{\partial z^{1}}+i \frac{\partial}{\partial w_{1}}, \cdots, \frac{\partial}{\partial z^{k}}+i \frac{\partial}{\partial w_{k}}, \frac{\partial}{\partial z^{k+1}}-i \frac{\partial}{\partial w_{k+1}}, \cdots, \frac{\partial}{\partial z^{n}}-i \frac{\partial}{\partial w_{n}}\right\} .
$$

The Gauß maps $L$ and $L^{\perp}$ induce Gauß maps

$$
\begin{array}{ll}
L_{M}: & M \rightarrow \Gamma \backslash G r_{0}^{k, l}\left(\mathbb{C}^{2 n}\right) \\
L_{M}^{\perp} & : \quad M \rightarrow \Gamma \backslash G r_{0}^{l, k}\left(\mathbb{C}^{2 n}\right)
\end{array}
$$

into the quotient of the Grassmannian by the holonomy group $\Gamma=\operatorname{Hol}(\nabla) \subset \operatorname{Sp}\left(\mathbb{R}^{2 n}\right)$ of the flat symplectic connection $\nabla$.

Corollary 2 (i) The dual Gauß map $L_{M}: M \rightarrow \Gamma \backslash G r_{0}^{k, l}\left(\mathbb{C}^{2 n}\right)$ of $M$ is holomorphic. (ii) The Gauß map $L_{M}^{\perp}: M \rightarrow \Gamma \backslash G r_{0}^{l, k}\left(\mathbb{C}^{2 n}\right)$ is antiholomorphic.

The Grassmannian $G r_{0}^{k, l}\left(\mathbb{C}^{2 n}\right)$ is a pseudo-Hermitian symmetric space and, in particular, a homogeneous pseudo-Kähler manifold. If $\Gamma \subset \operatorname{Sp}\left(\mathbb{R}^{2 n}\right)$ acts properly discontinuously on $G r_{0}^{k, l}\left(\mathbb{C}^{2 n}\right)$ then $\Gamma \backslash G r_{0}^{k, l}\left(\mathbb{C}^{2 n}\right)$ is a locally symmetric space of pseudo-Hermitian type.

\subsection{Holomorphic coordinates on the Grassmannian $G r_{0}^{k, l}\left(\mathbb{C}^{2 n}\right)$ of complex Lagrangian subspaces of signature $(k, l)$}

In this section we shall introduce a local model for the Grassmannian $G r_{0}^{k, l}\left(\mathbb{C}^{2 n}\right)$ and determine the corresponding local expression for the dual Gauß map. This model is a pseudo-Riemannian analogue of the Siegel upper half-space

$$
\operatorname{Sym}^{+}\left(\mathbb{C}^{n}\right):=\left\{A \in \operatorname{Mat}(n, \mathbb{C}) \mid A^{t}=A \text { and } \operatorname{Im} A \text { is positive definite }\right\} .
$$

Our aim is to construct holomorphic coordinates for the complex manifold $G r_{0}^{k, l}\left(\mathbb{C}^{2 n}\right)$ in a Zariski-open neighborhood of a point $W_{0}$ of the Grassmannian represented by a 
Lagrangian subspace $W_{0} \subset V$ of signature $(k, l)$. Using a transformation from $\operatorname{Sp}\left(\mathbb{R}^{2 n}\right)$ we can assume that $W_{0}=W_{o}$, see (4.4). Let $U_{0} \subset G r_{0}^{k, l}\left(\mathbb{C}^{2 n}\right)$ be the open subset consisting of $W \in G r_{0}^{k, l}\left(\mathbb{C}^{2 n}\right)$ such that the projection

$$
\pi_{(z)}: V=T^{*} \mathbb{C}^{n}=\mathbb{C}^{n} \oplus\left(\mathbb{C}^{n}\right)^{*} \rightarrow \mathbb{C}^{n}
$$

onto the first summand ( $z$-space) induces an isomorphism

$$
\left.\pi_{(z)}\right|_{W}: W \stackrel{\sim}{\rightarrow} \mathbb{C}^{n}
$$

Notice that $U_{0} \subset G r_{0}^{k, l}\left(\mathbb{C}^{2 n}\right)$ is an open neighborhood of the base point $W_{0}$. For elements $W \in U_{0}$ we can express $w_{i}$ as a function of $z=\left(z^{1}, \ldots, z^{n}\right)$. In fact,

$$
w_{i}=\sum C_{i j} z^{j}
$$

where

$$
\left(C_{i j}\right) \in \operatorname{Sym}_{k, l}\left(\mathbb{C}^{n}\right)=\left\{A \in \operatorname{Mat}(n, \mathbb{C}) \mid A^{t}=A \quad \text { and } \quad \operatorname{Im} A \text { has signature }(k, l)\right\} .
$$

\section{Proposition 7 The map}

$$
C: U_{0} \rightarrow \operatorname{Sym}_{k, l}\left(\mathbb{C}^{n}\right), \quad W \mapsto C(W):=\left(C_{i j}\right)
$$

is a local holomorphic chart for the Grassmannian $G r_{0}^{k, l}\left(\mathbb{C}^{2 n}\right)$.

Remark: The open subset $\operatorname{Sym}_{k, l}\left(\mathbb{C}^{n}\right) \subset \operatorname{Sym}\left(\mathbb{C}^{n}\right)=\left\{A \in \operatorname{Mat}(n, \mathbb{C}) \mid A^{t}=A\right\}$ is a generalization of the famous Siegel upper half-space $\operatorname{Sym}_{n, 0}\left(\mathbb{C}^{n}\right)=\operatorname{Sym}^{+}\left(\mathbb{C}^{n}\right)$, which is a Siegel domain of type I. In the latter case, we have $U_{0}=\operatorname{Sp}\left(\mathbb{R}^{2 n}\right) / \mathrm{U}(n)$ and a global coordinate chart

$$
C: G r_{0}^{n, 0}\left(\mathbb{C}^{2 n}\right)=\operatorname{Sp}\left(\mathbb{R}^{2 n}\right) / \mathrm{U}(n) \stackrel{\sim}{\rightarrow} \operatorname{Sym}_{n, 0}\left(\mathbb{C}^{n}\right)
$$

We shall now describe the dual Gauß map $L$ in local holomorphic coordinates in neighborhoods of $p_{0} \in \widetilde{M}$ and $L\left(p_{0}\right) \in G r_{0}^{k, l}\left(\mathbb{C}^{2 n}\right)$. Applying a transformation from $\operatorname{Sp}\left(\mathbb{R}^{2 n}\right)$, if necessary, we can assume that $L\left(p_{0}\right) \in U_{0}$. We put $U:=L^{-1}\left(U_{0}\right)$. The open subset $U \subset \widetilde{M}$ is a neighborhood of $p_{0}$.

Let $\phi: \widetilde{M} \rightarrow T^{*} \mathbb{C}^{n}$ be the Kählerian Lagrangian immersion. It defines a system of local (special) holomorphic coordinates

$$
\varphi:=\left.\pi_{(z)} \circ \phi\right|_{U}: U \stackrel{\sim}{\rightarrow} U^{\prime} \subset \mathbb{C}^{n}, \quad p \mapsto\left(z^{1}(\phi(p)), \cdots, z^{n}(\phi(p))\right)
$$

and we have the following commutative diagram

$$
\begin{array}{rll}
U & \stackrel{L}{\longrightarrow} & U_{0} \\
\varphi \downarrow & & \downarrow C \\
U^{\prime} & \stackrel{L_{U}}{\longrightarrow} & \operatorname{Sym}_{k, l}\left(\mathbb{C}^{n}\right)
\end{array}
$$


where the vertical arrows are holomorphic diffeomorphisms and $L_{U}$ at $z=\left(z^{1}, \ldots z^{n}\right)$ is given by

$$
L_{U}(z)=\left(F_{i j}(z)\right):=\left(\frac{\partial^{2} F(z)}{\partial z^{i} \partial z^{j}}\right)
$$

Here $F=F(z)$ is a holomorphic function on $U^{\prime} \subset \mathbb{C}^{n}$ determined, up to a constant, by the equations

$$
w_{j}(\phi(p))=\left.\frac{\partial F}{\partial z^{j}}\right|_{z(\phi(p))} .
$$

Summarizing, we obtain the following proposition.

Proposition 8 The dual Gauß map L has the following coordinate expression

$$
L_{U}=C \circ L \circ \varphi^{-1}=\left(F_{i j}\right) \text {, }
$$

where $\varphi: U \rightarrow \mathbb{C}^{n}$ is the (special) holomorphic chart of $\widetilde{M}$ associated to the Kählerian Lagrangian immersion $\phi$, see (4.14), and $C: U_{0} \rightarrow \operatorname{Sym}\left(\mathbb{C}^{n}\right)$ is the holomorphic chart of $G r_{0}^{k, l}\left(\mathbb{C}^{2 n}\right)$ constructed in (4.12).

\subsection{The special Kähler metric in affine coordinates}

As before, let $(M, J, g, \nabla)$ be a special Kähler manifold of Hermitian signature $(k, l)$, $k+l=n=\operatorname{dim}_{\mathbb{C}} M$, and $(\widetilde{M}, J, g, \nabla)$ its universal covering. As in section 1 , we shall now consider the metric $g$ in a $\nabla$-parallel frame. Such a frame is provided by the Kählerian Lagrangian immersion $\phi: \widetilde{M} \rightarrow V$. In fact, any point $p \in \widetilde{M}$ has a neighborhood in which the functions $\widetilde{x}^{i}:=\operatorname{Re} z^{i} \circ \phi, \widetilde{y}_{i}:=\operatorname{Re} w_{i} \circ \phi, i=1, \ldots, n$, form a system of local $\nabla$-affine coordinates. We recall that the $\nabla$-parallel Kähler form is given by $\omega=2 \sum d \widetilde{x}^{i} \wedge d \widetilde{y}_{i}$. This implies that the globally defined one-forms $\sqrt{2} d \widetilde{x}^{i}, \sqrt{2} d \widetilde{y}_{i}$ constitute a $\nabla$-parallel unimodular frame

$$
\left(e^{a}\right)_{a=1, \ldots, 2 n}=\left(e^{1}, \ldots, e^{2 n}\right):=\left(\sqrt{2} d \widetilde{x}^{1}, \ldots, \sqrt{2} d \widetilde{x}^{n}, \sqrt{2} d \widetilde{y}_{1}, \ldots, \sqrt{2} d \widetilde{y}_{n}\right)
$$

of $T^{*} \widetilde{M}$ with respect to the metric volume form $\nu=(-1)^{n+1} \frac{\omega^{n}}{n !}=2^{n} d \widetilde{x}^{1} \wedge \ldots \wedge d \widetilde{y}_{n}$. The dual frame $\left(e_{a}\right)$ of $T \widetilde{M}$ is also $\nabla$-parallel and unimodular. The metric defines a smooth map

$$
G: \widetilde{M} \rightarrow \operatorname{Sym}_{2 k, 2 l}^{1}\left(\mathbb{R}^{2 n}\right)=\left\{A \in \operatorname{Mat}(2 n, \mathbb{R}) \mid A^{t}=A, \operatorname{det} A=1 \text { has signature }(2 k, 2 l)\right\}
$$

by

$$
p \mapsto G(p):=\left(g_{a b}(p)\right):=\left(g_{p}\left(e_{a}, e_{b}\right)\right) .
$$

We will call $G=\left(g_{a b}\right)$ the fundamental matrix of $\phi$. As before, we identify

$$
\operatorname{Sym}_{2 k, 2 l}^{1}\left(\mathbb{R}^{2 n}\right)=\operatorname{SL}(2 n, \mathbb{R}) / \mathrm{SO}(2 k, 2 l) \text {. }
$$

This is a pseudo-Riemannian symmetric space. For conventional reasons, in this section, $\mathrm{SO}(2 k, 2 l) \subset \mathrm{SL}(2 n, \mathbb{R})$ is defined as the stabilizer of the symmetric matrix

$$
E_{o}:=\operatorname{diag}\left(\mathbb{1}_{k},-\mathbb{1}_{l}, \mathbb{1}_{k},-\mathbb{1}_{l}\right)
$$


The fundamental matrix induces a map

$$
G_{M}: M \rightarrow \Gamma \backslash \operatorname{Sym}_{2 k, 2 l}^{1}\left(\mathbb{R}^{2 n}\right)
$$

into the quotient of $\operatorname{Sym}_{2 k, 2 l}^{1}\left(\mathbb{R}^{2 n}\right)$ by the action of the holonomy group $\Gamma=\operatorname{Hol}(\nabla) \subset$ $\operatorname{Sp}\left(\mathbb{R}^{2 n}\right) \subset \mathrm{SL}(2 n, \mathbb{R})$. The target $\Gamma \backslash \operatorname{Sym}_{2 k, 2 l}^{1}\left(\mathbb{R}^{2 n}\right)$ is a pseudo-Riemannian locally symmetric space, provided that $\Gamma$ acts properly discontinuously.

Theorem 4 The fundamental matrix $G: \widetilde{M} \rightarrow \operatorname{Sym}_{2 k, 2 l}^{1}\left(\mathbb{R}^{2 n}\right)=\operatorname{SL}(2 n, \mathbb{R}) / \operatorname{SO}(2 k, 2 l)$ takes values in the totally geodesic submanifold

$$
\iota: G r_{0}^{k, l}\left(\mathbb{C}^{2 n}\right)=\mathrm{Sp}\left(\mathbb{R}^{2 n}\right) / \mathrm{U}(k, l) \hookrightarrow \mathrm{SL}(2 n, \mathbb{R}) / \mathrm{SO}(2 k, 2 l)
$$

and coincides with the dual Gauß map $L: \widetilde{M} \rightarrow G r_{0}^{k, l}\left(\mathbb{C}^{2 n}\right): G=\iota \circ L$.

Proof: The proof will follow from a geometric description of the inclusion $\iota$. To any Lagrangian subspace $W \in G r_{0}^{k, l}\left(\mathbb{C}^{2 n}\right)$ we can associate the scalar product $g^{W}:=\left.\operatorname{Re} \gamma\right|_{W}$ of signature $(2 k, 2 l)$ on $W \subset V$. The projection onto the real points

$$
\operatorname{Re}: V=T^{*} \mathbb{C}^{n} \rightarrow T^{*} \mathbb{R}^{n}=\mathbb{R}^{2 n}, \quad v \mapsto \operatorname{Re} v=\frac{1}{2}(v+\bar{v})
$$

induces an isomorphism of real vector spaces $W \stackrel{\sim}{\rightarrow} \mathbb{R}^{2 n}$ the inverse of which we denote by $\psi=\psi_{W}$. We claim that

$$
\iota(W)=\psi^{*} g^{W}=:\left(g_{a b}^{W}\right)=: G^{W} .
$$

To check this, it is sufficient to prove that the map

$$
G r_{0}^{k, l}\left(\mathbb{C}^{2 n}\right) \ni W \mapsto G^{W} \in \operatorname{Sym}_{2 k, 2 l}^{1}\left(\mathbb{R}^{2 n}\right)
$$

is $\operatorname{Sp}\left(\mathbb{R}^{2 n}\right)$-equivariant and maps the base point $W_{o}$ with stabilizer $\mathrm{U}(k, l)$, see (4.4), to the base point $E_{o}$ with stabilizer $\mathrm{SO}(2 k, 2 l)$, see $(4.23)$. Let us verify that indeed $G^{W_{o}}=E_{o}$.

Using the definition of $\gamma$, one finds for the basis

$$
\left(e_{j}^{ \pm}\right):=\left(\frac{\partial}{\partial z^{j}} \pm i \frac{\partial}{\partial w_{j}}\right)
$$

of $V$ that the only non-vanishing components of $\gamma$ are $\gamma\left(e_{j}^{ \pm}, e_{j}^{ \pm}\right)= \pm 2$. This shows that $g^{W_{o}}=\left.\operatorname{Re} \gamma\right|_{W_{o}}$ is represented by the matrix $2 E_{o}$ with respect to the basis

$$
\left(e_{1}^{+}, \ldots, e_{k}^{+}, e_{1}^{-}, \ldots, e_{l}^{-}, i e_{1}^{+}, \ldots, i e_{k}^{+}, i e_{1}^{-}, \ldots, i e_{l}^{-}\right) .
$$

In order to calculate $G^{W_{o}}=\left(g_{a b}^{W_{o}}\right)=\left(g\left(\psi e_{a}, \psi e_{b}\right)\right)$, we need to pass from the real basis (4.30) of $W_{o}$ to the real basis $\left(\psi e_{a}\right)$.

Recall that the real structure $\tau$ is complex conjugation with respect to the coordinates $\left(z^{i}, w_{i}\right)$. This implies that

$$
\begin{aligned}
& \psi^{-1}\left(e_{j}^{+}\right)=\frac{\partial}{\partial x^{j}}=\sqrt{2} e_{j}, \quad \psi^{-1}\left(i e_{j}^{+}\right)=-\frac{\partial}{\partial y_{j}}=-\sqrt{2} e_{n+j}, j=1, \ldots, k, \\
& \psi^{-1}\left(e_{j}^{-}\right)=\frac{\partial}{\partial x^{j}}=\sqrt{2} e_{j}, \quad \psi^{-1}\left(i e_{j}^{-}\right)=\frac{\partial}{\partial y_{j}}=\sqrt{2} e_{n+j}, \quad j=1, \ldots, l .
\end{aligned}
$$


This shows that $G^{W_{o}}=E_{o}$.

It remains to check the equivariance of $W \mapsto G^{W}=\psi^{*} g$. Using the definition of the map $\psi=\psi_{W}: \mathbb{R}^{2 n} \rightarrow W$, one easily checks that, under the action of $\Lambda \in \operatorname{Sp}\left(\mathbb{R}^{2 n}\right), \psi$ transforms as

$$
\psi_{\Lambda W}=\left.\Lambda \circ \psi_{W} \circ \Lambda^{-1}\right|_{\mathbb{R}^{2 n}} .
$$

From this we deduce the transformation law of $G^{W}$ :

$$
G^{\Lambda W}=\psi_{\Lambda W}^{*} g^{\Lambda W}=\left(\Lambda^{-1}\right)^{*} \psi_{W}^{*} \Lambda^{*} g^{\Lambda W}=\left(\Lambda^{-1}\right)^{*} \psi_{W}^{*} g^{W}=\left(\Lambda^{-1}\right)^{*} G^{W}=\Lambda \cdot G^{W} .
$$

The above claim (4.27), together with the fact that

$$
g^{L(p)}=g_{p} \quad \text { and } \quad G^{L(p)}=G(p)
$$

for all $p \in \widetilde{M}$, implies that

$$
\iota(L(p))=G^{L(p)}=G(p) .
$$

Corollary 3 The fundamental matrix $G: \widetilde{M} \rightarrow \operatorname{Sym}_{2 k, 2 l}^{1}\left(\mathbb{R}^{2 n}\right)$ is pluriharmonic.

Proof: $G=\iota \circ L$ is the composition of the holomorphic map $L: \widetilde{M} \rightarrow G r_{0}^{k, l}\left(\mathbb{C}^{2 n}\right)$ with the totally geodesic inclusion $G r_{0}^{k, l}\left(\mathbb{C}^{2 n}\right) \subset \operatorname{Sym}_{2 k, 2 l}^{1}\left(\mathbb{R}^{2 n}\right)$. The composition of a holomorphic map with a totally geodesic map is pluriharmonic.

\section{References}

[ACD] D. V. Alekseevsky, V. Cortés and C. Devchand, Special complex manifolds, J. Geom. Phys. 42 (2002), 85-105.

[CV] S. Cecotti , C. Vafa, Topological-antitopological fusion, Nuclear Physics B 367 (1991), 351-461.

[D] B. Dubrovin, Geometry and integrability of topological-antitopological fusion, Commun. Math. Phys. 152 (1992) 539-564.

[F] D.S. Freed, Special Kähler manifolds, Commun. Math. Phys. 203 (1999), no. 1, $31-52$.

[H] C. Hertling, $t t^{*}$-Geometry, Frobenius manifolds, their connections, and the construction for singularities, J. Reine Angew. Math. 555 (2003), 77-161.

[KN] S. Kobayashi, K. Nomizu, Foundations of differential geometry, volume II, Interscience Publishers, 1969.

[S1] L. Schäfer, $t t^{*}$-Geometrie und pluriharmonische Abbildungen, Diplomarbeit an der Universität Bonn, December 2002.

[S2] L. Schäfer, tt*-bundles and pluriharmonic maps, to appear. 